\title{
The Pathophysiology of Cellulite: Can the Puzzle Eventually Be Solved?*
}

\section{Ilja Kruglikov}

Wellcomet GmbH, Karlsruhe, Germany.

Email: i.kruglikov@wellcomet.de

Received November $4^{\text {th }}, 2011$; revised November $20^{\text {th }}, 2011$; accepted December $1^{\text {st }}, 2011$

\begin{abstract}
The pathophysiology of cellulite is still largely unknown. Here, we propose a new pathophysiology that connects the development of cellulite with several newly-discovered hallmarks of white adipose tissue. According to this theory, cellulite appears in hypertrophic fat tissue that is associated with overproduction of low molecular weight hyaluronan (HA). This can induce different types of fibrosis, producing inhomogeneous spatial tension in the tissue. This pathophysiology serves to explain the most well known peculiarities of cellulite appearance as well as to formulate the theoretically optimal cellulite treatment strategy.
\end{abstract}

Keywords: Cellulite; Pathophysiology; Adipose Fibrosis; Hyaluronan; Optimal Treatment Strategy

\section{Introduction}

Cellulite is currently considered to be an aesthetic condition that is thought to affect more than $85 \%$ of women over the age of 20 . It appears most frequently in the gluteofemoral and abdominal regions. The effectiveness of any known treatment method is very limited and the results are generally short-termed. The main problem is that the pathophysiology of cellulite remains largely unknown and this substantially restricts the development of optimal treatment strategies.

Several theories of cellulite pathophysiology have been formulated during recent years. Their main ideas are reflected in the different names given to describe cellulite, e.g. nodule liposclerosis, oedematose fibrosclerotic panniculopathy, gynoid lipodystrophy, dermatomyoliposclerosis, etc. These theories can be subdivided into three groups, each of which has different anatomical or physicological changes in the cellulite tissue as a primary effect:

- An increase in water content as the concentration of proteoglycans' rises [1], leading to the development of an oedema;

- A change in the local microcirculation [2,3], with the progressive development of lymphoedema, lipoedema or even lipolymphoedema;

- An abnormal arrangement of collagen structures in the tissue [4] and/or compartmentation of the fat tissue through fibrose septae.

${ }^{*}$ Conflict of interests (COI): PhD, Dr. Sci. Ilja Kruglikov is the managing partner of Wellcomet $\mathrm{GmbH}$, Karlsruhe.
The pathophysiological steps of cellulite development were described in [5]. They include, amongst other phenomena: the alteration of metabolic reactions in interstitial matrix; damage to collagen fibres; an accumulation of free water; a reduction in the number of proteoglycans and of HA concentration; alterations in connective structures and the collagen system; development of lipoedema and lipolymphoedema; disorders in the lipogenesis-lipolysis system; and alterations in venous-lymphatic microcirculation. Some of these steps clearly contradict the above theories.

Each new treatment method that has been developed during recent decades has been mainly dedicated to one of these pathophysiological ideas. So, the drainage methods were primarily applied to reduce the free water content in cellulite fat tissue. Vacuum massage and ultrasound were applied in different forms to modulate the pressure distribution in the subcutaneous tissue and, consequently, to improve its microcirculation. RF current was claimed to reorient the collagen structures, which are temperature sensitive. Long-term pressure application through elastocompression should also reorient the collagen structures. All these methods as well as different combinations thereof (e.g. RF with ultrasound, vacuum with RF-current, IR-light and so on) could not significantly improve results over a reasonable period of time.

One possible explanation of this phenomenon could be that the existing pathophysiological theories of cellulite are either false or incomplete, and so an important link that could lead to a breakthrough has not yet been dis- 
covered. None of these pathophysiological theories can really answer the specific questions of cellulite development and appearance such as:

1) Why does cellulite mainly appear in the gluteofemoral region?

2) Why can women with a normal body mass index (BMI) develop cellulite?

3) Why can the same treatments given to women with normal and high BMI values often show very different results?

4) Why does cellulite react to different physical (and some pharmacological) treatment methods?

5) Why are treatment results generally relatively shorttermed and why do patients sometimes develop a resistance to the same method of therapy as treatment progresses?

6) Why do cellulite rates vary significantly between different ethnic groups, e.g. Asian-Indians and Caucasians?

In this article we describe the new pathophysiology of cellulite, which will not only answer these questions, but also bring the aforementioned theories together. This pathophysiology is based on new results that have been obtained from research into obesity during the last several years. These concern fat tissue turnover, the relationship between hypertrophy and hyperplasia in a fat tissue, the role of HA in a fat tissue as well as adipose tissue fibrosis as a new hallmark of pathologic (hypertrophic) fat tissue.

\section{Regional Fat Depots}

The most important, classical dogmas of fat tissue, which directly or indirectly influence the pathophysiological theories of the cellulite, are:

- Every regional fat depot consists of a homogeneous population of adipocytes;

- There is no adipocyte turnover in adults;

- Water and collagen contents in a fat tissue are low.

All these dogmas are nowadays considered to be wrong and need to be replaced, which has a very deep impact not only on the general theory of fat tissue, but also on the pathophysiology of cellulite.

First of all, adipocytes from different regional fat depots have bi-modal size distributions [6], representing two populations of small and big cells. This seems to be typical for fat tissue and immediately raises the question of whether there is a mixture of small and big adipocytes, with specific weighting of these two cell types in different fat depots and under different conditions? This is not only a very important pathophysiological feature, but can also strongly influence the treatment of cellulite, making single treatment strategies very questionable.

Indeed, it was demonstrated that fat depots, even in people with the same BMI values, can be of two qualitatively different types [7]: hypertrophic (with low cellularity and big adipocytes) and hyperplastic (with high cellularity and small adipocytes). Adipocytes have a renewal rate of approximately $10 \%$ per year, i.e. it will take approximately 10 years to fully renew the fat tissue. Together with the current knowledge that the amount of adipocytes produced is genetically predetermined, this means that even after their removal, the number of fat cells can be completely renewed. Although this turnover rate is low, the total amount of renewed adipocytes per year can be estimated to be between four and 12 billion [7]. This renewal will lead to the remodelling of extracellular matrix along with the simultaneous modulation of its collagen and water content. Fat tissue structure can, thus, be significantly changed in just one year. Whilst the degree of cellulite can remain unchanged for much longer than one year, this phenomenon must be resistant against remodelling of the fat tissue or be tightly connected with this process.

This new picture we have gained of fat tissue raises the next questions:

- Do different regional fat depots have different size distributions of adipocytes?

- Which of the two types of adipocytes is more important in cellulite and how can the status of the mixture be determined?

- What is, theoretically, the best method of fat reduction? Is it the reduction of cellularity through damage and/or removal of adipocytes, or the reduction of cell volumes through lipolysis activation?

BMI alone cannot be used to predict the type of the fat tissue as well as the adipocytes' size distribution. In people of a normal BMI, the biggest adipocytes are located in the femoral region, followed by the gluteal and abdominal regions [8]. The average size of adipocytes increases with an increased BMI, whereas the difference between distinct regional fat depots diminishes progresssively. Something that could be especially important in the field of cellulite research is that some women with a normal BMI $<25$ have adipocytes of a size comparable with the average size of adipocytes in women with a BMI $>30$. Additionally, there is a pronounced hyperplasia in the gluteofemoral region of women with an increased BMI (cellularity almost doubles between the groups of subjects with $\mathrm{BMI}<25$ and $\mathrm{BMI}>30$ ). Although the biggest adipocytes are located in the gluteofemoral region and their number increases with BMI, hypertrophy can generally appear in people with different BMIs and even in lean subjects.

Could the hallmark of cellulite appearance be hypertrophic adipocytes developing a special, extracellular structure, which makes the pattern of such fat tissue clearly different from a hyperplastic one? To analyse this 
problem, we have to discuss the peculiarities of at least two additional structural components of the fat tissuewater and collagen.

\section{Water in a Fat Tissue}

Water content in a normal fat tissue is, indeed, less than $20 \%$, but it can vary significantly in different types of fat as well as regionally. Most of this water is located extracellular $(14 \%-16 \%)$ and only $2 \%-6 \%$ intra-cellular. These values can change dramatically in hypertrophic fat tissue. Water accumulation in such tissue is primarily connected with a significantly increased concentration of HA $(40 \%-50 \%$ in extracellular space and up to four times that in pericellular space) [9], which will cause the amount of water that accumulates to be much more than that in normal fat tissue. Such water content seems to correlate with the HA-bounding capacity of collagen type VI [10], which is one of the main collagen types in fat tissue. Consequently, the water in such tissue exists primarily in a gel form and must be clearly differentiated from oedema, which is the accumulation of free water. For this reason, the development of an oedema cannot be one of the early pathophysiological steps of cellulite development because this can only occur after saturation of the HA gel, the swelling of which is dependent on the level of HA concentration and can be tremendous. This contradicts the current theory of cellulite development [5], which considers a reduction in HA and free water accumulation to be early steps of cellulite pathophysiology.

What could be the reason for high HA production in hypertrophic fat tissue? High HA concentration is a hallmark of metabolically active tissue as well as of proliferative tissue. Very high concentration of HA is recorded in the growth area of solid tumours, e.g. concentration of HA in liposarcoma can be 50 - 200 times that of lipoma or of non-neoplastic adipose tissue [11]. This water accumulation is needed for the cell expansion and proliferation as well as, probably, for the viability of the cells to be protected. A relatively moderate increase of HA concentration in hypertrophic fat tissue can be connected with the expansion of adipocytes in a non-rigid environment.

The progressive hypertrophy of adipocytes will lead to the further accumulation of HA and bound water in the tissue, increasing the swelling of the gel and its total turgor. Uncontrolled increase of the cell volume can lead to the rupture of adipocytes' membranes. To prevent damage, some mechanism that can effectively restrict the adipocytes progression and further water accumulation is needed. One defence mechanism could be the elimination of AQP7 channels in expanding adipocytes. These channels are responsible for both water and glycerol ex- change and their internalisation will block the lipolysis, rendering the adipocytes "metabolically blind" [12]. Another defence mechanism would be the production of a rigid environment around adipocytes, which could mechanically restrict their expansion.

\section{Adipose Tissue Fibrosis}

Two important discoveries of the last years force us to completely re-analyse the role of collagen in fat tissue. First of all, collagen content changes dramatically as adipocytes move from the "commitment" phase into the growth phase, connected with storage of a high concentration of triglycerides [13]. There are not only quantitative, but also qualitative differences in collagen structure between normal and hypertrophic fat tissues - the latter has a pronounced amount of collagen types IV and VI, which together build a special collagen net of non-fibrillar nature. It is well known that an absence of collagen VI in fat tissue leads to a strong hypertrophy of adipocytes $[14,15]$ and that the concentration of this collagen defines the degree of fat hypertrophy. Secondly, it was shown that adipose tissue fibrosis is a general hallmark of metabolically changed adipocytes, which is needed to restrict their further expansion $[15,16]$.

This fibrosis can appear in two different forms. $F \boldsymbol{i}$ brillar fibrosis consists mainly of collagen types I and III, has the form of thick collagen bundles [16] and can penetrate the fat tissue in different directions. The thicker the bundles, the greater the tension in surrounding fat tissue. This type of fibrosis looks similar to local scarring. Pericellular fibrosis is predominantly of the laminar type. It consists of the basal lamina (collagen IV) and microfibrils of the extracellular matrix (collagen VI). Collagen VI content greatly increases with rising BMI [17,18]. Whereas the total fibrotic volume in subcutis is almost independent of BMI [16], as BMI rises, the pericellular fibrosis increases by three to seven times. This clearly demonstrates the importance of this collagen in different types of adipose pathologies. Pericellular fibrosis restricts the adipocytes' expansion, but at the same time produces local tension, which can lead to inflammation [15]. At first glance, this last phenomenon appears to be very unusual, because, until now, there was a widespread belief that large adipocytes, rather than small ones, are responsible for the negative metabolic development of fat tissue (e.g. for insulin intolerance). Now it looks like the fibrosis, and not the fat cells themselves, is responsible for this negative metabolic feature.

Fibrotic structures can be developed not only around adipocytes, but also around blood vessels [16]. Such a development will lead to vasoconstriction, consequently disturbing the microcirculation and maybe also the lymph transport. This oft-described feature of cellulite, 
which in different pathophysiological theories was considered to be a prime reason of its development, is, thus, not a reason, but a consequence of adipose tissue fibrosis. It looks like the adipocytes develop the special extracellular matrix structure around them to put themselves "under arrest".

\section{Correlation between HA Distribution and Adipose Tissue Fibrosis}

There seems to be a remarkable correlation between space distributions of HA and fibrosis structures in hypertrophic fat tissue. HA is not homogeneously distributed between adipocytes, but has two specific spatial patterns - cable-form and pericellular [19,20], which are similar to the fibrillar and pericellular configurations of fibrosis. The HA cables can be long and span many cell lengths.

HAs of different molecular weighting have very different functions and are now considered to be the informational substances [21]. HA found in hypertrophic fat tissue has a molecular weight of approximately $15 \mathrm{kDa}$ or less [9]. It is known that low molecular weight HA fragments can significantly stimulate production of collagen types I and III [22,23], promoting tissue fibrosis. It can be speculated that intercellular cable-form and pericellular HA have different molecular weights and can, therefore, stimulate production of different collagens, e.g. of type I and III intercellular, and IV and VI pericellular. Subsequently, the local production of low molecular weight HA fragments should be the reason for the local tissue fibrosis (scarring) and, consequently, for the appearance of cellulite.

\section{Brief Formulation of the New Cellulite Pathophysiology}

Cellulite is a visual skin appearance that reflects the processes of adipose tissue fibrosis in a hypertrophic fat tissue, which are the hallmarks of pathological alteration of human, white adipose tissue that is connected with production of low molecular weight HA fragments.

Different steps of this pathophysiology are summarized in Figure 1.

Now we can try to answer some important questions concerning the development of cellulite.

Why does cellulite mainly appear in the gluteofemoral region? Why do some women with a normal BMI have cellulite in the femoral-and almost never in the abdominal region?

Adipocytes' size distributions in gluteofemoral and abdominal regions are different [8]. People with BMI $<25$ have $0.57 \pm 0.23 \mu \mathrm{g}$ TG per cell in femoral, $0.48 \pm 0.15$ $\mu \mathrm{g}$ TG in gluteal and only $0.41 \pm 0.20 \mu \mathrm{g}$ TG in abdominal regions. In people with BMI $>30$ these values are
$0.83 \pm 0.18 \mu \mathrm{g}, 0.71 \pm 0.23 \mu \mathrm{g}$ und $0.78 \pm 0.24 \mu \mathrm{g} \mathrm{TG}$ per adipocyte, respectively. We see that some women with a normal BMI have femoral adipocytes of a size comparable with the average size of these cells in women with obesity. The abdominal adipocytes in lean women are small and cannot produce fibrosis and the consequent cellulite appearance in this region. This enables us to estimate the critical size of adipocytes for cellulite appearance to be approximately $70-75 \mu \mathrm{m}$. One can estimate that under this condition, approximately $20 \%-25 \%$ of lean women will develop cellulite in the femoral region.

Why do the same treatments often show different results in subjects with normal and high BMI values?

Adipose tissue fibrosis consists of two different components-fibrillar fibrosis (mainly of collagen types I and III) and pericellular fibrosis (mainly of collagen types IV and VI). These collagens are the substrates of different matrix metalloproteinases (MMPs) and not all of them can be destroyed with one and the same treatment method. If people with different BMIs have different weighting of these two types of fibrosis, the treatment results have to be dependent on BMI. This really does look to be the case- -whereas the percentage of total fibrosis in white adipose tissue is the same in lean and obese subjects, the percentage of pericellular fibrosis rises tremendously with increasing BMI [16].

Why does cellulite react to different physical (as well as to some pharmacological) treatment methods?

If the hallmark of cellulite is adipose tissue fibrosis in hypertrophic fat tissue, it has to react to all the physical and pharmacological methods that can modify its fibrotic structure. This modification can be caused by modulation of specific MMPs. Such modulation is known to occur in the treatment of mild hyperthermia (e.g. through RFcurrent or light applications), massage, elastocompression or windings and ultrasound as well as in the longterm stimulation with electrical current. However, the degree of modulation is very dependent on the parameters of the chosen treatment method. Normal pharmacological substances applied topically cannot penetrate deep enough into the fat tissue and so they only show results in a form of skin camouflage. The situation can be changed through the injection of substances that can significantly modulate the collagen types I and III (e.g. through the injection of collagenases) or IV (by injecting gelatinases). In some respects, this makes the treatment of cellulite similar to the treatment of hyper- trophic and keloid scars.

Why are the treatment results generally relatively short-termed and why can subjects develop a resistance to the same method of therapy as treatment progresses?

An effective cellulite treatment has to be able to re- 


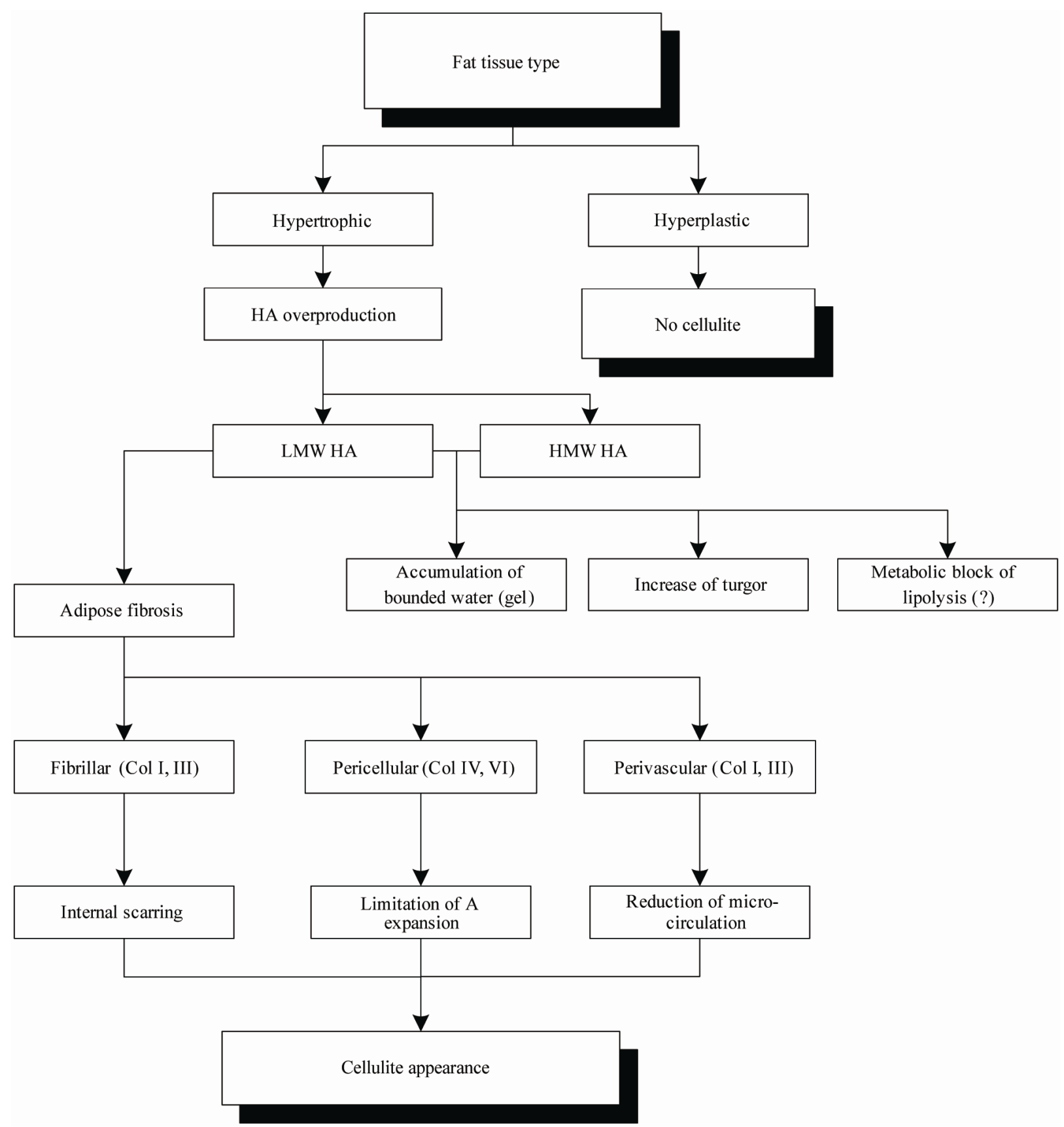

Figure 1. The pathophysiology of cellulite. Abbreviations: LMW HA-low molecular weight HA; HMW HA-high molecular weight HA; A-adipocyte.

duce the fibrosis and, consequently, the mechanical tension in the tissue. If nothing happens to the adipocytes during this treatment, they will have no mechanical restriction and expand further. Such additional expansion will immediately trigger the production of new fibrotic structures. This domino effect demonstrates that cellulite cannot be effectively treated with any one stand-alone method (even with the simultaneous combination of different treatments in one application) and the results can only be optimised through a special treatment strategy.
Why do cellulite rates vary significantly between different ethnic groups, e.g. Asian-Indians and Caucasians?

Fibrosis of the fat tissue correlates negatively with adipocytes' size. Women of the Asian-Indian ethnic group have a much higher content of collagen VI in the fat tissue than Caucasian women [16]. Thus, this ethnic group already has significantly higher pericellular fibrosis in the early stages of the adipocytes' expansion. Adipocytes cannot increase their volumes in a rigid environment in 
the same way they can in a loose tissue [14]. Consequently, they remain relatively small, the hypertrophy will not develop and cellulite does not occur. On the other hand, the Asian-Indians compensate for this with higher fat tissue fibrosis and consequent higher inflammation in the fat tissue, which means a higher risk of diabetes.

\section{Are Different Cellulite Theories Only Reflect the Adipose Tissue Fibrosis?}

\subsection{Theories Connected with Water Accumulation and Vasoconstriction}

Concentration of proteoglycans and, consequently, water retention in cellulite is significantly increased [1]. This fact led to the formulation of the theory that interstitial osmotic pressure in a cellulite tissue must be of an increased value, which in turn causes the vasoconstriction of the vessels, hypoxia and local oedema production [2, 3].

Increased HA content in a hypertrophic fat tissue leads to accumulation of bound water. Normally, this water cannot be as easily removed through the creation of an oedema alone - this water has high viscosity and has to first be transformed into free water. An additional problem for water evacuation could be the above-mentioned fibrotic structures around the blood vessels [16]. This perivessel fibrosis consists primarily of collagen type I and can construct rigid cases of low elasticity around the vessels, reducing their dilation and, consequently, the water exchange in the tissue, which appears as a microcirculation disturbance.

\subsection{Theories Connected with the Pathological Collagen Structure of the Fat Tissue}

Compartmentation and a unique orientation of collagen bundles in fat tissue are often considered to be the main reasons for cellulite. According to the cellulite pathophysiology discussed in this article, such a compartmentation is the consequence of cell hypertrophy and tissue fibrosis. The observed clustering of adipocytes can be the result of sub-atmospheric pressure in a fat tissue, which presses the cells to each other. Fibrosis of the fat tissue is induced by low molecular weight HA, the overproduction of which is a key factor and whose regulation could be a possible goal in the field of cellulite treatment.

\section{The Theoretically Optimal Cellulite Treatment Strategy}

A theoretically optimal cellulite treatment strategy has to consist of at least three steps:

Step 1: Destruction of fibrosis in fat tissue to reduce internal tension;
Step 2: Stimulation of lipolysis to reduce the volumes of hypertrophic adipocytes;

Step 3: Production of new collagen to prevent de novo expansion of adipocytes.

Step 1 concerns both types of adipose fibrosis-fibrillar and pericellular with different types of collagen involved. Such destruction cannot be realised through simple modulation of one MMP (e.g. MMP-1 for collagen I) and needs a complex activation procedure or combination of different treatment methods. Step 2 can be realised through minimal invasive (e.g. mesotherapy) or some special non-invasive local treatments. However, it has to be taken into account that hypertrophic adipocytes can also be metabolically inactive, which can require water removal from the fat tissue as a pre-activation step [12]. New collagen production, which is needed for de novo fibrosis of the fat tissue with decreased in size adipocytes, can be realised through various processes (e.g. by inducing local hyperthermia), but this supposes the simultaneous suppression of MMP-production to prevent the immediate cleavage of new produced collagen.

\section{Conclusion}

The new pathophysiology formulated in this article considers the overproduction of low molecular weight HA fragments in a hypertrophic fat tissue to be a primary step of cellulite development. These fragments induce different types of adipose tissue fibrosis, which produce inhomogeneous tension in the tissue. This can reasonably explain the main features of cellulite appearance and can be used to formulate the theoretically optimal treatment strategy.

\section{REFERENCES}

[1] T. Lotti, M. D. Ghersetich, C. Grappone and G. Dini, "Proteoglycans in So-Called Cellulite," International Journal of Dermatology, Vol. 29, No. 4, 1990, pp. 272274. doi:10.1111/j.1365-4362.1990.tb02560.x

[2] S. B. Curri, "Cellulite and Fatty Tissue Microcirculation," Cosmetics \& Toiletries, Vol. 108, 1993, pp. 51-58.

[3] A. B. Rossi and A. L. Vergnanini, "Cellulite: A Review," Journal of the European Academy of Dermatology and Venereology, Vol. 14, No. 4, 2000, pp. 251-262. doi:10.1046/j.1468-3083.2000.00016.x

[4] B. Querleux, C. Cornillon, O. Jolivet and J. Bittoun, "Anatomy and Physiology of Subcutaneous Adipose Tissue by in Vivo Magnetic Resonance Imaging and Spectroscopy: Relationships with Sex and Presence of Cellulite," Skin Research \& Technology, Vol. 8, No. 2, 2002, pp. 118-124. doi:10.1034/j.1600-0846.2002.00331.x

[5] P. A. Bacci and G. Leibaschoff, "Pathophysiology of Cellulite," In: M. P. Goldman, P. A. Bacci, G. Leibaschoff, D. Hexel and F. Angelini, Eds., Cellulite Pathophysiology and treatment, Taylor \& Francis Group, New York, 
2006, pp. 41-74.

[6] M. Jernas, J. Palming, K. Sjöholm, E. Jennische, P.-A. Svensson, B. G. Gabrielsson, M. Levin, A. Sjörgen, M. Rudemo, T. C. Lystig, B. Carlsson, L. M. S. Carlsson and M. Lönn, "Separation of Human Adipocytes by Size: Hypertrophic Fat Cells Display Distinct Gene Expression," FASEB Journal, Vol. 20, No. 9, 2006, pp. E832E839. doi:10.1096/fj.05-5678fje

[7] E. Arner, P. Westermark, K. L. Spalding, T. Britton, M. Ryden, J. Frisen, S. Bernard and P. Arner, "Adipocyte Turnover: Relevance to Human Adipose Tissue Morphology," Diabetes, Vol. 59, No. 1, 2010, pp. 105-109. doi:10.2337/db09-0942

[8] Y. D. Tchoukalova, C. Koutsari, M. V. Karpyak, S. B. Votruba, E. Wendland and M. D. Jensen, "Subcutaneous Adipocytes Size and Body Fat Distribution," American Journal of Clinical Nutrition, Vol. 87, 2008, pp. 56-63.

[9] C. Y. Han, S. Subramanian, C. K. Chan, M. Omer, T. Chiba, T. N. Wight and A. Chait, "Adipocyte-Derived Serum Amyloid A3 and Hyaluronan Play a Role in Monocyte Recruitment and Adhesion," Diabetes, Vol. 56, No. 9, 2007, pp. 2260-2273. doi:10.2337/db07-0218

[10] C. M. Kielty, S. P. Whittaker, M. E. Grant and C. A. Shuttleworth, "Type VI Collagen Microfibrills: Evidence for a Structural Association with Hyaluronan," Journal of Cell Biology, Vol. 118, No. 4, 1992, pp. 979-990. doi:10.1083/jcb.118.4.979

[11] M. Sobue, K. Miura, K. Kataoka, K. Tsuji and J. Takeuchi, "Glycosaminoglycan-Synethetic Activity of Neoplastic and Non-Neoplastic Adipose Tissue," British Journal of Cancer, Vol. 42, 1980, pp. 477-480. doi:10.1038/bjc.1980.261

[12] I. Kruglikov and K. Hoffmann, "Nicht Invasive Umfangreduzierung. Warum Bleiben Einige Fettablagerungen Behandlungsresistent?" Kosmetische Medizin, No. 3, 2011, pp. 108-113.

[13] E. C. M. Mariman and P. Wang, "Adipocyte Extracellular Matrix Composition, Dynamics and Role in Obesity," Cellular and Molecular Life Sciences, Vol. 67, No. 8, 2010, pp. 1277-1292. doi:10.1007/s00018-010-0263-4

[14] T.-H. Chun, K. B. Hotary, F. Sabeh, A. R. Saltiel, E. D. Allen and S. J. Weiss, "A Pericellular Collagenase Directs the 3-Dimensional Development of White Adipose Tissue," Cell, Vol. 125, No. 3, 2006, pp. 577-591. doi:10.1016/j.cell.2006.02.050

[15] T. Khan, E. S. Muise, P. Iyengar, Z. V. Wang, M. Chandalia, N. Abate, B. B. Zhang, P. Bonaldo, S. Chua and P.
E. Scherer, "Metabolic Dysregulation and Adipose Tissue Fibrosis: Role of Collagen VI," Molecular and Cellular Biology, Vol. 29, No.6, 2009, pp. 1575-1591. doi:10.1128/MCB.01300-08

[16] A. Divoux, J. Tordjman, D. Lacasa, N. Veyrie, D. Hugol, A. Aissat, A. Basdevant, M. Guerre-Millo, C. Poitou, J.-D. Zucker, P. Bedossa and K. Clement, "Fibrosis in Human Adipose Tissue: Composition, Distribution, and Link with Lipid Metabolism and Fat Mass Loss," Diabetes, Vol. 59, No. 11, 2010, pp. 2817-2825. doi: $10.2337 / \mathrm{db} 10-0585$

[17] I. Nakajima, S. Muroya, R.-I. Tanabe and K. Chikuni, "Extracellular Matrix Development during Differentiation into Adipocytes with a Unique Increase in Type V and VI Collagen," Biology of the Cell, Vol. 94, No. 3, 2002, pp. 197-203. doi:10.1016/S0248-4900(02)01189-9

[18] M. Pasarica, B. Gowronska-Kozak, D. Burk, I. Remedios, D. Hymel, J. Gimble, E. Ravussin, G. A. Bray and S. R. Smith, "Adipose Tissue Collagen VI in Obesity," The Journal of Clinical Endocrinology \& Metabolism, Vol. 94, No. 12, 2009, pp. 5155-5162. doi:10.1210/jc.2009-0947

[19] S. P. Evanko, M. I. Tammi, R. H. Tammi and T. N. Wight, "Hyaluronan-Dependent Pericellular Matrix," Advanced Drug Delivery Reviews, Vol. 59, No. 13, 2007, pp. 13511365. doi:10.1016/j.addr.2007.08.008

[20] C. A. de la Motte and J. A. Drazba, "Viewing Hyaluronan: Imaging Contributes to Imagining New Roles for This Amazing Matrix Polymer," Journal of Histochemistry and Cytochemistry, Vol. 59, No. 3, 2011, pp. 252-257. doi: $10.1369 / 0022155410397760$

[21] R. Stern, A. A. Asari and K. N. Sugahara, "Hyaluronan Fragments: An Information-Rich System," European Journal of Cell Biology, Vol. 85, No. 8, 2006, pp. 699715. doi:10.1016/j.ejcb.2006.05.009

[22] Y. Li, M. Rahmanian, C. Widström, G. Lepperdinger, G. I. Frost and P. Heldin, "Irradiation-Induced Expression of Hyaluronan (HA) Synthase 2 and Hyaluronidase 2 Genes in Rat Lung Tissue Accompanies Active Turnover of HA and Induction of Types I and III Collagen Gene Expression," American Journal of Respiratory Cell and Molecular Biology, Vol. 23, 2000, pp. 411-418.

[23] T. S. Wilkinson, S. Potter-Perigo, C. Tsoi, L. C. Altman and T. N. Wight, "Pro- and Anti-Inflammatory Factors Cooperate to Control Hyaluronan Synthesis in Lung Fibroblasts," American Journal of Respiratory Cell and Molecular Biology, Vol. 31, No. 1, 2004, pp. 92-99. doi:10.1165/rcmb.2003-03800C 